Algebraic 85 Geometric Topology

Volume 1 (2001) 491-502

Published: 10 September 2001

ATG

\title{
The product formula for Lusternik-Schnirelmann category
}

\author{
Joseph RoITBERG
}

\begin{abstract}
If $C=C_{\phi}$ denotes the mapping cone of an essential phantom map $\phi$ from the suspension of the Eilenberg-Mac Lane complex $K=$ $K(\mathbb{Z}, 5)$ to the 4 -sphere $S=S^{4}$, we derive the following properties: (1) The LS category of the product of $C$ with any $n$-sphere $S^{n}$ is equal to 3 ; (2) The LS category of the product of $C$ with itself is equal to 3 , hence is strictly less than twice the LS category of $C$. These properties came to light in the course of an unsuccessful attempt to find, for each positive integer $m$, an example of a pair of 1-connected CW-complexes of finite type in the same Mislin (localization) genus with LS categories $m$ and $2 m$. If $\phi$ is such that its $p$-localizations are inessential for all primes $p$, then by the main result of [10], the pair $C_{*}=S \vee \Sigma^{2} K, C$ provides such an example in the case $m=1$.
\end{abstract}

\section{AMS Classification 55M30}

Keywords Phantom map, Mislin (localization) genus, Lusternik-Schnirelmann category, Hopf invariant, Cuplength

\section{Introduction}

In this sequel to [10], we record two additional curious properties of the space $X$ described in the main Theorem of [10].

Recall that $X$ is the mapping cone of an essential map $\phi$ from $\Sigma K$, the suspension of the Eilenberg-Mac Lane complex $K=K(\mathbb{Z}, 5)$, to $S=S^{4}$, the 4 -sphere. Henceforth we use the notation $C=C_{\phi}$ for this space. Recall from $[10]$ that $\phi$ factors uniquely as

$$
\phi=\psi \circ r
$$

where

$$
r: \Sigma K \longrightarrow S_{(0)}^{6}
$$

is a rationalization map and

$$
\psi: S_{(0)}^{6} \longrightarrow S
$$


Both $\phi$ and $\psi$ are phantom maps in the sense of [14] (see also [9, End of section 1]); they become inessential when restricted to finite subcomplexes of their respective domains. We study $D=D_{\psi}$, the mapping cone of $\psi$, side by side with $C$. The space $D$ is simpler (it is finite-dimensional) and less interesting (it does not have finite type) than $C$ and serves as a model for the latter. The two results below apply to both spaces; the proofs in the (easier) case of $D$ provide motivation and guidance for the proofs in the case of $C$.

The first result states, in view of the main Theorem of [10], that the spaces $C, D$ satisfy the Ganea "conjecture" for all spheres. Thus:

Theorem 1.1 For any $n>0$,

$$
\operatorname{cat}\left(C \times S^{n}\right)=3=\operatorname{cat}\left(D \times S^{n}\right) .
$$

As an application of Theorem 1.1, we obtain a pair of spaces in the same Mislin genus having LS categories 2 and 3 . Namely, if $C_{*}$ (respectively $D_{*}$ ) denotes the mapping cone of the trivial map with domain $\Sigma K$ (respectively $S_{(0)}^{6}$ ), and if $\phi$ is a special phantom map as in [10, Example 2] (that is the $p$-localization of $\phi$ is inessential for every prime $p$ ), then the spaces $C \times S^{n}, C_{*} \times S^{n}$ are in the same Mislin genus, as are the spaces $D \times S^{n}, D_{*} \times S^{n}$, and

$$
\begin{array}{ll}
\operatorname{cat}\left(C \times S^{n}\right)=3, & \operatorname{cat}\left(C_{*} \times S^{n}\right)=2 ; \\
\operatorname{cat}\left(D \times S^{n}\right)=3, & \operatorname{cat}\left(D_{*} \times S^{n}\right)=2 .
\end{array}
$$

This application is only moderately interesting since we may generalize Example 2 of [10] to get an example of a pair of spaces in the same Mislin genus having LS categories $m$ and $m+1$ for any $m>0$. Indeed, we need only replace $K$ by $K(\mathbb{Z}, 4 m+1)$ and $S$ by $\mathbb{H} P^{m}$, quaternionic projective $m$-space, and argue as in [10], applying appropriate results of Iwase [8]; details are omitted. What would be more interesting is to find a pair of spaces in the same Mislin genus with LS categories $m$ and $2 m$ for any $m>0$. For according to a result of Cornea [1], with improvement by Stanley [11] (see also Félix-Halperin-Thomas [3]),

$$
\operatorname{cat}(X) \leq 2 \cdot \sup _{p}\left(\operatorname{cat}\left(X_{(p)}\right)\right),
$$

where $X$ is a 1 -connected CW-complex of finite type and $X_{(p)}$ is its $p$-localization.

Examples of the type just mentioned would show that the inequality (1.1) is sharp for all $m>0$. To that end, our original thought was to try the $m$-fold product spaces $C_{*}^{m}, C^{m}$ (or $D_{*}^{m}, D^{m}$ ). However, this attempt fails, as the next result shows. 
Theorem $1.2 \operatorname{cat}(C \times C)=3=\operatorname{cat}(D \times D)$.

Thus $C$ is a 1-connected $\mathrm{CW}$-complex of finite type such that $\operatorname{cat}(C \times C)$ is strictly smaller than $2 \cdot \operatorname{cat}(C)$. Examples of strict inequality in the product formula $\operatorname{cat}(A \times B) \leq \operatorname{cat}(A)+\operatorname{cat}(B)$ go back to Fox [4]. However, the two factors in Fox's example are distinct spaces: they are Moore spaces $S^{2} \cup_{p}$ $e^{3}, S^{2} \cup_{q} e^{3}$ with respect to distinct primes $p$ and $q$. See also Ganea-Hilton [5] for a generalization of Fox's example as well as the observation that $\operatorname{cat}(K(\mathbb{Q}, 1) \times$ $K(\mathbb{Q}, 1)) \leq 3$ (which is strictly smaller than $2 \cdot \operatorname{cat}(K(\mathbb{Q}, 1))=4$ ); of course, $K(\mathbb{Q}, 1)$, unlike $C$, is neither 1 -connected nor of finite type. An alternate derivation of the inequality $\operatorname{cat}(K(\mathbb{Q}, 1) \times K(\mathbb{Q}, 1)) \leq 3$ is possible along the lines of the first part of the proof below of Theorem 1.2.

A more recent example of strict inequality in the product formula, similar in flavor to Fox's example (in both situations, $A \vee B$ is homotopy equivalent to $A \times B$ ), is contained in Félix-Halperin-Lemaire [2]. In this example, $A$ is a 1-connected $\mathrm{CW}$-complex whose (reduced) homology groups are all finite and $B$ is a 1-connected, rational $\mathrm{CW}$-complex. The main result of [2] states that - unlike the situation when $A, B$ are both $K(\mathbb{Q}, 1)-\operatorname{cat}(A \times B)=\operatorname{cat}(A)+$ $\operatorname{cat}(B)$ whenever $A, B$ are rationalizations of 1 -connected $\mathrm{CW}$-complexes of finite type.

The landscape changed radically when Iwase [7] discovered examples of strict inequality in the product formula in which one of the factors (but not both!) is a sphere. Systematic approaches to Ganea's "conjecture", with many more examples, have been developed since then; see [8], [11], Vandembroucq [13], Stanley [12] and Harper [6].

We remark that the examples in [6] and [12], illustrating the strict inequality $\operatorname{cat}(A \times A)<2 \cdot \operatorname{cat}(A)$ for $A 1$-connected of finite type are quite different from those in Theorem 1.2. In each of Harper's examples, $A$ is the mapping cone of a suitable map from a sphere to another sphere and $\operatorname{cat}(A)=2$, $\operatorname{cat}(A \times A) \leq 3$. In Stanley's example, $A$ is the mapping cone of a suitable map from a sphere to a bouquet of two spheres and $\operatorname{cat}(A)=2=\operatorname{cat}(A \times A)$. In contrast to the situation in Theorem 1.1, the examples in [6] and [12] do not satisfy the Ganea "conjecture". In fact, $\operatorname{cat}\left(A \times S^{n}\right)=2$ for all $n \geq 2$ in two of the three examples in [6] and cat $\left(A \times S^{n}\right)=2$ for all $n \geq 1$ in the third example in [6] and in the example in [12].

The proofs of Theorems 1.1 and 1.2 are contained in the next section. The proof of Theorem 1.1 builds on the proof of the main Theorem of [10], bringing in more of the Hopf invariant technology of [8]. 
We also take the opportunity to correct a mis-statement in the last paragraph on p. 99 of [10]; I thank Jianzhong Pan for bringing the error to my attention.

As for the proof of Theorem 1.2, the inequalities

$$
\operatorname{cat}(C \times C) \leq 3, \quad \operatorname{cat}(D \times D) \leq 3
$$

follow from classical obstruction theory arguments. Similar, but slightly more elaborate, arguments lead to the inequalities

$$
\operatorname{cat}\left(C^{k}\right) \leq k+1, \quad \operatorname{cat}\left(D^{k}\right) \leq k+1
$$

for $3 \leq k \leq 6$ as well, but these arguments break down for $k=7$. To establish the opposite inequalities

$$
\operatorname{cat}(C \times C) \geq 3, \quad \operatorname{cat}(D \times D) \geq 3,
$$

we exploit techniques of [8] together with cuplength arguments. In fact, we show that $D \times D$ has cuplength 3 . This latter fact fails for $C \times C$ (whose cuplength is 2). To remedy this failure, we introduce a notion of refined cup products - which in the case of $C \times C$ consists of a blend of ordinary cohomology and cohomotopy - and show that $C \times C$ has non-zero refined cup products of length 3 , thereby leading to the desired inequality.

The latter approach also leads to an alternate, albeit less direct, proof of Theorem 1.1, as well as of the main Theorem of [10].

\section{Proofs}

Before giving the proof of Theorem 1.1, which closely follows the lines of the proof of the main Theorem of [10], we wish to point out that throughout the last paragraph on page 99 of [10], the space $S_{(0)}^{6}$ should be replaced by $\Sigma K$. To see that the argument beginning in that paragraph and concluding on page 100 is valid for $\Sigma K$, it is only necessary to observe that we may identify the map

$$
\left[\Sigma K, S^{7}\right] \longrightarrow\left[\Sigma K, E^{2}\left(\Omega S^{4}\right)\right]
$$

induced by the inclusion of the bottom $S^{7}$ into $E^{2}\left(\Omega S^{4}\right)$ with the analogous map

$$
\left[S_{(0)}^{6}, S^{7}\right] \longrightarrow\left[S_{(0)}^{6}, E^{2}\left(\Omega S^{4}\right)\right]
$$


denoted in [10] by $\beta_{1}$; here $E^{2}\left(\Omega S^{4}\right)$ is a space homotopy equivalent to the 2 -fold join $\Omega S^{4} * \Omega S^{4}$. Indeed, we have a commutative diagram

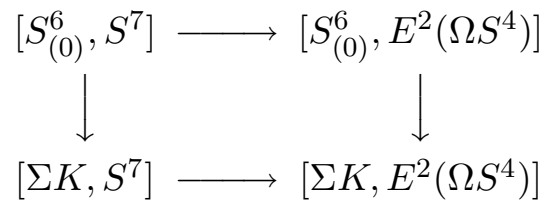

where the vertical maps are induced by rationalization $r: \Sigma K \rightarrow S_{(0)}^{6}$ and are isomorphisms; see [10, (2.5)].

We now give the proof of Theorem 1.1.

Proof of Theorem 1.1 It is clear that

$$
\operatorname{cat}\left(A \times S^{m}\right) \leq 3, \quad A=C \text { or } D,
$$

for any $m \geq 1$. To prove that

$$
\operatorname{cat}\left(A \times S^{m}\right)=3 \text {, }
$$

we appeal to a special case of $[8$, Theorem $3.9(2)]$ according to which it suffices to establish the following variant of (iii) on page 98 of [10]:

$$
\left(E^{2}(\Omega i) * j_{m-1}\right) \circ \Sigma^{m} H_{1}(\alpha)
$$

is essential, where the left-most map is the join of the map induced by the inclusion $i: S \subset A$ with the canonical map $j_{m-1}: S^{m-1} \rightarrow \Omega S^{m}, \alpha=\phi$ ( respectively $\psi$ ) if $A=C$ (respectively $D$ ), and $H_{1}(\alpha)$ is the Berstein-HiltonHopf invariant of $\alpha$ as generalized by Iwase ([8, Definition 2.4]) and Stanley ([11, Definition 3.4]).

Now the argument on page 99 of [10] prior to the last paragraph actually shows that $\Sigma^{m} H_{1}(\alpha)$ is essential for any $m \geq 0$. Moreover the subsequent argument (suitably amended; see above) shows that $E^{2}(\Omega i)$ has a left homotopy inverse. Since $\Sigma j_{m-1}$ has a left homotopy inverse, it follows that $E^{2}(\Omega i) * j_{m-1}$ too has a left homotopy inverse, and (iii') is verified.

In the case $A=D$, it is even permissible to "ignore" the map $E^{2}(\Omega i) * j_{m-1}$; indeed, that map induces a monomorphism from $\left[S_{(0)}^{6} * S^{m-1}, E^{2}\left(\Omega S^{4}\right) * S^{m-1}\right]$ to $\left[S_{(0)}^{6} * S^{m-1}, E^{2}(\Omega D) * \Omega S^{m}\right]$ since $S_{(0)}^{6}$ has dimension 7 . See also [8, Remark $3.10]$.

Remark Since any rationalization map $r: \Sigma K \rightarrow S_{(0)}^{6}$ is a suspension, it follows from [8, Proposition 2.11(1)] that

$$
H_{1}(\varphi)=H_{1}(\psi) \circ r .
$$

We next give the proof of Theorem 1.2. 
Proof of Theorem 1.2 To prove

$$
\operatorname{cat}(B) \leq 3, \quad B=C \times C \text { or } D \times D,
$$

it suffices to show that the Ganea fibration

$$
P^{3}(\Omega B) \longrightarrow B,
$$

with fiber

$$
E^{4}(\Omega B) \simeq \Omega B * \Omega B * \Omega B * \Omega B,
$$

admits a section; see [8, Theorem 2.1]. For either value of $B$ in (2.1), we easily verify that $E^{4}(\Omega B)$ is 14-connected - more precisely, $E^{4}(\Omega B)$ has the homotopy type of a $\mathrm{CW}$-complex of the form

$$
\left(S^{15} \vee \cdots \vee S^{15}\right) \cup\{\text { cells of dimension } \geq 18\} .
$$

Next observe that

$$
H_{n}(D \times D)=0
$$

for $n>14$; note that unless the coefficients are explicitly indicated, homology and cohomology groups are understood to be integral. Thus

$$
H^{n}\left(D \times D ; \pi_{n-1}\left(E^{4}(\Omega(D \times D))\right)=0\right.
$$

for all $n$. Since the obstructions to a section of $(2.2)$ in the case $B=D \times D$ lie in these cohomology groups, it is clear that a section exists.

Two remarks:

(1) An alternate approach to the existence of a section of (2.2) in this case, but not in the case $B=C \times C$, is via a slight variant of [8, Theorem 5.5] - details are omitted.

(2) We do not assert that there is a unique section of (2.2) in the case $B=$ $D \times D$. In fact, the (vertical homotopy classes of) sections are classified by

$$
H^{15}\left(D \times D ; \pi_{15}\left(E^{4}(\Omega(D \times D))\right) \approx \operatorname{Ext}(\mathbb{Q}, \mathbb{Z} \oplus \cdots \oplus \mathbb{Z}),\right.
$$

which has the cardinality of the continuum.

To deal with the case $B=C \times C$, first note that a homology decomposition of $K$, say

$$
S^{5}=K[5] \subset \cdots \subset K[m] \subset \cdots \subset K,
$$

where each $K[m]$ is a finite complex, naturally induces a homology decomposition of $C$,

$$
S=C[4] \subset \cdots \subset C[m] \subset \cdots \subset C,
$$


where each $C[m]$ is likewise a finite complex. Explicitly, we take $C[m]$ to be the mapping cone of the restriction of $\phi$ to $\Sigma K[m-1]$. Thus $C \times C$ is the ascending union of the finite subcomplexes $C[m] \times C[m]$, about which we make two claims:

(1) $\operatorname{cat}(C[m] \times C[m])=2, m \geq 4$. In fact, the homotopy equivalence $C[m] \simeq$ $S \vee \Sigma^{2} K[m-1], m \geq 4$, resulting from the fact that $\phi$ is phantom, implies that $\operatorname{cat}(C[m])=1$, hence that $\operatorname{cat}(C[m] \times C[m])=2, m \geq 4$.

(2) For each $m, H_{n}(C[m] \times C[m])$ is finite for all $n>14$. This follows immediately from the fact that $H_{n}(C \times C)$ is finite for all $n>14$.

It follows from (1) that for each $m$, the inclusion $C[m] \times C[m] \subset C \times C$ lifts to the total space $P^{3}(\Omega(C \times C))$ of the fibration (2.2). Moreover, the number of such lifts is finite since, thanks to (2), the cohomology groups $H^{n}(C[m] \times$ $C[m] ; \pi_{n}\left(E^{4}(\Omega(C \times C))\right)$ are finite for all $n$. A variant of the classical argument utilized in the first paragraph of page 100 of [10] then leads to the conclusion that (2.2) admits a section in the case $B=C \times C$.

We now verify that

$$
\operatorname{cat}(B) \geq 3, \quad B=C \times C \text { or } D \times D,
$$

beginning with the case $B=D \times D$. Our strategy is to detect a non-zero 3 -fold cup product in the integral cohomology ring of $D \times D$. More precisely, if $u$ and $v$ are the canonical generators (up to sign) of $H^{4}(D \times D)$, we show that

$$
u^{2} v \neq 0 \text {. }
$$

We first prove the weaker statement

$$
u^{2} \neq 0
$$

and then generalize the proof of (2.4) to obtain (2.3). Let $u_{*}$ be the restriction of $u$ to $D \times * \subset D \times D$. To prove (2.4), it suffices to prove

$$
u_{*}^{2} \neq 0 \text {. }
$$

Applying [8, Theorem 4.1, Remark 4.3] to the cofibration sequence

$$
S_{(0)}^{6} \longrightarrow S \longrightarrow D
$$

we infer that the reduced diagonal map

$$
\Delta^{\prime}=\Delta_{D}^{\prime}: D \longrightarrow D \wedge D
$$

factors as the composite

$$
(i \wedge i) \circ(e \wedge e) \circ \Sigma H_{1}(\psi) \circ q: D \longrightarrow D \wedge D,
$$

where 
(1) $q: D \rightarrow D / S=S_{(0)}^{7}$ is the collapsing map,

(2) $\Sigma H_{1}(\psi): S_{(0)}^{7} \rightarrow \Sigma E^{2}(\Omega S) \simeq \Sigma \Omega S \wedge \Sigma \Omega S$ is the suspension of the Berstein-Hilton-Hopf invariant of $\psi$, and

(3) $(i \wedge i) \circ(e \wedge e)$ is induced by the evaluation map $e: \Sigma \Omega S \rightarrow S$ and the inclusion map $i: S \subset D$.

Abbreviating $K(m)=K(\mathbb{Z}, m)$ and viewing $u_{*}$ as (the homotopy class of) a map

$$
u_{*}: D \longrightarrow K(4),
$$

we may view $u_{*}^{2}$ as the composite

$$
\mu \circ\left(u_{*} \wedge u_{*}\right) \circ \Delta^{\prime}: D \longrightarrow D \wedge D \longrightarrow K(4) \wedge K(4) \longrightarrow K(8),
$$

where $\mu$ represents a generator of $H^{8}(K(4) \wedge K(4))$. From (2.6) and (2.7), we see that $u_{*}^{2}$ may be represented as the composite

$$
\mu \circ(j \wedge j) \circ(e \wedge e) \circ \Sigma H_{1}(\psi) \circ q: D \longrightarrow K(8),
$$

where $j \wedge j$ is induced by a generator $j$ of $H^{4}(S)$ while the other maps in the composite have been defined above.

Reasoning as in the proof of $[10,(2.3)]$ shows that $(e \wedge e) \circ \Sigma H_{1}(\psi)$ is non-zero. It is then routine to argue that the composite (2.8) is itself non-zero. Thus (2.5), and with it (2.4), is proved.

Next let $u_{\bullet}, v_{\bullet}$ be the images of $u, v$ under the cohomology map induced by the inclusion $D \times S \subset D \times D$. Of course, the restriction of $u_{\bullet}$ to $D \times * \subset D \times S$ is just $u_{*}$. To prove (2.3), it suffices to prove

$$
u_{\bullet}^{2} v_{\bullet} \neq 0 .
$$

To that end, we apply [8, Corollary 4.1.1] to the cofibration sequence

$$
S_{(0)}^{10} \longrightarrow(D \times *) \cup(S \times S) \rightarrow D \times S
$$

to infer that the reduced diagonal map

$$
\Delta^{\prime} \wedge 1_{S}=q^{\prime} \circ\left(\Delta^{\prime} \times 1_{S}\right): D \times S \rightarrow(D \wedge D) \times S \longrightarrow D \wedge D \wedge S,
$$

where $q^{\prime}$ is the collapsing map, factors as

$$
\left(i \wedge i \wedge 1_{S}\right) \circ\left(e \wedge e \wedge 1_{S}\right) \circ \Sigma^{5} H_{1}(\psi) \circ q^{!}: D \times S \longrightarrow D \wedge D \wedge S
$$

where

(1) $q^{!}: D \times S \rightarrow D \times S /(D \times *) \cup(S \times S)=(D / S) \wedge S$ is the collapsing map, 
(2) $D \times S /(D \times *) \cup(S \times S)=(D / S) \wedge S=S_{(0)}^{11}$,

(3) $\Sigma^{5} H_{1}(\psi): S_{(0)}^{11} \rightarrow \Sigma \Omega S \wedge \Sigma \Omega S \wedge S$ is the 5 -fold iterated suspension of $H_{1}(\psi)$,

(4) $e \wedge e \wedge 1_{S}: \Sigma \Omega S \wedge \Sigma \Omega S \wedge S \rightarrow S \wedge S \wedge S$ is induced by $e$, and

(5) $i \wedge i \wedge 1_{S}: S \wedge S \wedge S \rightarrow D \wedge D \wedge S$ is induced by $i$.

From (2.10), we see that $u_{\bullet}^{2} v_{\bullet}$ may be represented as the composite

$$
\nu \circ(j \wedge j \wedge j) \circ\left(e \wedge e \wedge 1_{S}\right) \circ \Sigma^{5} H_{1}(\psi) \circ q^{!}: D \times S \longrightarrow K(12)
$$

where $\nu$ represents a generator of $H^{12}(K(4) \wedge K(4) \wedge K(4))$.

Arguing as with (2.8), we readily verify that the composite (2.11) is non-zero. Thus (2.9), and with it (2.3), is proved.

Finally, we adapt the preceding argument to prove the inequality

$$
\operatorname{cat}(C \times C) \geq 3 .
$$

If $u, v$ now stand for the canonical generators (up to sign) of $H^{4}(C \times C)$, the analogs of (2.3) and (2.4) both fail. In fact, as the cohomology of $C$ is indistinguishable from that of $C_{*}$, the cuplength of $C$ is precisely 1 and the cuplength of $C \times C$ is precisely 2 . One might try replacing cohomology by cohomotopy since $\pi^{8}(C)=\left[C, S^{8}\right]\left(\right.$ as also $\pi^{8}(D)=\left[D, S^{8}\right] \approx H^{8}(D)$ ) turns out to be set-theoretically equivalent to $\operatorname{Ext}(\mathbb{Q}, \mathbb{Z})$ as noted in Lemma 2.1 below. Unfortunately, the Puppe sequence

$$
[\Sigma K, S] \longleftarrow[S, S] \longleftarrow[C, S] \longleftarrow\left[\Sigma^{2} K, S\right]
$$

associated to the cofibration sequence $\Sigma K \rightarrow S \rightarrow C$ gives that

$$
\pi^{4}(C)=[C, S]=0
$$

since the left-most map in (2.12) sends $[S, S]$ monomorphically to the (infinite cyclic) group generated by $\phi$, and $\left[\Sigma^{2} K, S\right]=0$ by [14, Theorem D] (see also [9, Theorem 4.2]). In other words, the cohomology classes $u, v$ are not compressible into $S$.

While neither cohomology alone nor cohomotopy alone supports a cuplength argument necessary to establish the inequality cat $(C \times C) \geq 3$, the following compromise between cohomology and cohomotopy provides a way out of the dilemma. Fix a CW-model for $K(m)$ such that the $m$-skeleton is $S^{m}$ and the $N$-skeleton $K(m)_{N}$ has only finitely many cells for all $N \geq m$. 
Lemma 2.1 (1) For $N \geq 9$, the inclusions $K(4)_{N} \subset K(4)_{N+1} \subset K(4)$ induce a commutative diagram

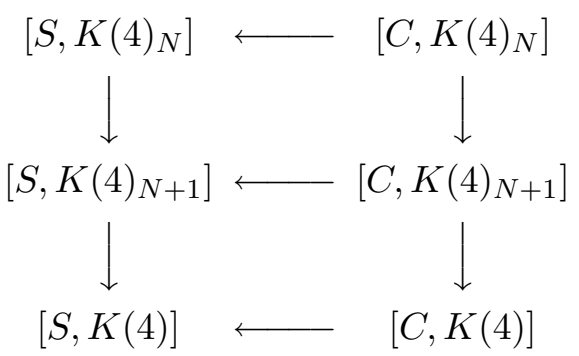

with each of the sets in (2.13) equivalent to $\mathbb{Z}$, and each of the arrows a bijection;

(2) For $N \geq 8$, the inclusion $K(8)_{N} \subset K(8)_{N+1}$ induces a commutative diagram

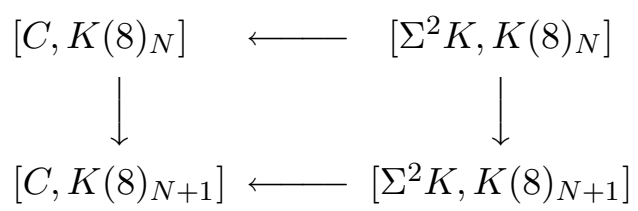

with each of the sets in (2.14) equivalent to $\operatorname{Ext}(\mathbb{Q}, \mathbb{Z})$, and each of the arrows a bijection;

(3) For $N \geq 12$, the inclusion $K(12)_{N} \subset K(12)_{N+1}$ induces a commutative diagram

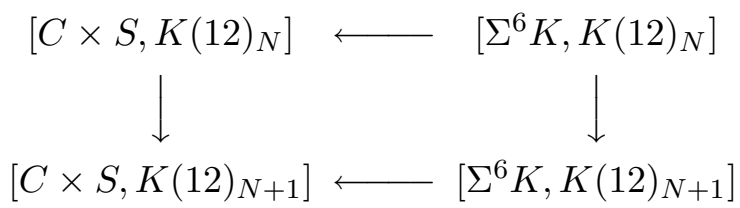

with each of the sets in (2.15) equivalent to $\operatorname{Ext}(\mathbb{Q}, \mathbb{Z})$, and each of the arrows a bijection.

Proof To prove the first part of (1), consider the commutative diagram with exact rows

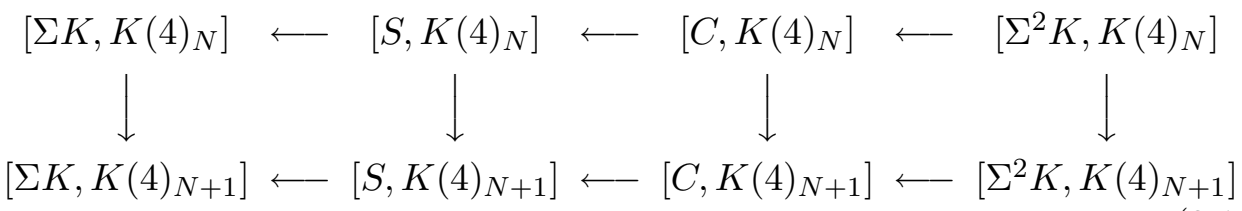

Algebraic $6 \mathcal{G}$ Geometric Topology, Volume 1 (2001) 
induced by the cofibration sequence $\Sigma K \rightarrow S \rightarrow C$. Notice that if $M \geq 9$,

$$
\pi_{7}\left(K(4)_{M}\right)=0=\pi_{8}\left(K(4)_{M}\right) .
$$

Therefore, by [14, Theorem D] (see also [9, Theorem 4.2]),

$$
\left[\Sigma K, K(4)_{M}\right]=0=\left[\Sigma^{2} K, K(4)_{M}\right]
$$

and (2.16) collapses to the upper square in (2.13). The proof of the rest of (1), and also (2) and (3), are similar. Observe that the diagram in (3) is induced by the cofibration sequence

$$
\Sigma^{5} K \longrightarrow(C \times *) \cup(S \times S) \longrightarrow C \times S .
$$

Define $u_{* N}, u_{\bullet N}, v_{\bullet N}$ to be the unique compressions of $u_{*}, u_{\bullet}, v_{\bullet}$ into $K(4)_{N}$, $N \geq 9$, guaranteed by part (1) of Lemma 2.1. Further, define two maps

$$
\mu_{N}: K(4)_{N} \wedge K(4)_{N} \longrightarrow K(8)_{2 N+1},
$$

and

$$
\nu_{N}: K(4)_{N} \wedge K(4)_{N} \wedge K(4)_{N} \longrightarrow K(12)_{3 N+1}
$$

as the maps uniquely determined by $\mu, \nu$. It may be assumed that these maps are cellular. We define the refined cup square $u_{* N}^{2}$ as the element represented by the composite

$$
\mu_{N} \circ\left(u_{* N} \wedge u_{* N}\right) \circ \Delta_{C}^{\prime}: C \longrightarrow C \wedge C \longrightarrow K(4)_{N} \wedge K(4)_{N} \longrightarrow K(8)_{2 N+1} .
$$

We define the refined 3-fold cup product, $u_{\bullet N}^{2} v_{\bullet N}$ similarly, using $\nu_{N}$. Observe that $u_{* N}^{2}$ compresses uniquely into $S^{8}$ by part (2) of Lemma 2.1. In other words, $u_{* N}$ is essentially a 4 -dimensional cohomology class while $u_{* N}^{2}$ is essentially an 8 -dimensional cohomotopy class. Similarly, by part (3) of Lemma 2.1, $u_{\bullet N}^{2} v_{\bullet N}$ is essentially a $12-$ dimensional cohomotopy class.

We now invoke the analog of (2.8) with $D$ replaced by $C, S_{(0)}^{7}$ replaced by $\Sigma^{2} K$ and $K(m)$ replaced by $K(m)_{N}, N \geq 9$, to conclude, with the help of Lemma 2.1 , that

$$
u_{* N}^{2} \neq 0,
$$

that is the refined cuplength of $C$ is at least 2. Moreover, arguing with the appropriate analog of (2.11), we find, once again with the help of Lemma 2.1, that

$$
u_{\bullet N}^{2} v \bullet N \neq 0
$$

that is the refined cuplength of $C \times C$ is at least 3 .

The classical argument showing that cuplength is a lower bound for cat readily generalizes to show that refined cuplength is also a lower bound for cat. Hence $\operatorname{cat}(C \times C)$ is at least 3, completing the proof of Theorem 1.2. 
This research was supported in part by a grant from the City University of New York PSC-CUNY Research Award Program.

\section{References}

[1] O. Cornea, Lusternik-Schnirelmann-categorical sections, Annales Scientifiques de l'École Normale Supérieure, $4^{e}$ Série, 28 (1995), 689-704.

[2] Y. Félix, S. Halperin and J.-M. Lemaire, The rational LS category of products and of Poincare duality complexes, Topology 37 (1998), 749-756.

[3] Y. Félix, S. Halperin and J.-C. Thomas, Lusternik-Schnirelmann category of skeleta. Preprint.

[4] R.H. Fox, On the Lusternik-Schnirelmann category, Annals of Mathematics 42 (1941), 333-370.

[5] T. Ganea and P. J. Hilton, On the decomposition of spaces in Cartesian products and unions, Proceedings Cambridge Philosophical Society 55 (1959), 248-256.

[6] J. Harper, Category and products. Preprint.

[7] N. Iwase, Ganea's conjecture on Lusternik-Schnirelmann category, Bulletin London Mathematical Society 30 (1998), 623-634.

[8] N. Iwase, $A_{\infty}$ method in Lusternik-Schnirelmann category. Preprint.

[9] J. Roitberg, Computing homotopy classes of phantom maps. CRM Proceedings and Lecture Notes, vol. 6 (1994), 141-168.

[10] J. Roitberg, The Lusternik-Schnirelmann category of certain infinite CWcomplexes, Topology 39 (2000), 95-101.

[11] D. Stanley, Spaces with Lusternik-Schnirelmann category $n$ and cone length $n+1$, Topology 39 (2000), 985-1019.

[12] D. Stanley, On the Lusternik-Schnirelmann category of maps. Preprint.

[13] L. Vandembroucq, Suspension des fibrations de Ganea et invariant de Hopf, Thèse, Université de Lille (1998).

[14] A. Zabrodsky, On phantom maps and a theorem of H. Miller, Israel Journal of Mathematics 58 (1957), 129-143.

Department of Mathematics and Statistics

Hunter College, CUNY

695 Park Avenue, New York, NY 10021, USA

Email: roitberg@math.hunter.cuny.edu

Received: 26 October 2000 Revised: 7 May 2001

Algebraic 83 Geometric Topology, Volume 1 (2001) 